\title{
Squamous cell carcinoma of the rectum: Practice trends and patient survival
}

\author{
Sunil W. Dutta ${ }^{1}$ (i) | Clayton E. Alonso ${ }^{1} \quad$ Mark R. Waddle ${ }^{2}$ | \\ Shiv R. Khandelwal ${ }^{1}$ | Einsley-Marie Janowski $^{1} \quad$ | Daniel M. Trifiletti ${ }^{2}$ (C)
}

${ }^{1}$ Department of Radiation Oncology, University of Virginia, Charlottesville, Virginia

${ }^{2}$ Department of Radiation Oncology, Mayo Clinic, Jacksonville, Florida

\section{Correspondence}

Sunil W. Dutta, Department of Radiation Oncology, University of Virginia,

Charlottesville, VA.

Email:nwdutta@gmail.com

\begin{abstract}
Purpose: Leverage the National Cancer Database (NCDB) to evaluate trends in management of nonmetastatic squamous cell cancer (SCC) of the rectum and their effect on survival for this uncommon tumor.

Methods and Materials: Retrospective data was obtained from the NCDB for patients diagnosed with SCC of the rectum between 2004 and 2014, including cT1-4, cN0-2, cM0 tumors (cohort A, $\mathrm{n}=2296$ ). A subgroup analysis was performed on locally advanced tumors (cT1-T2, N+ or cT3, N any, subcohort $\mathrm{B}, \mathrm{n}=883$ ), treated with chemoradiation $(n=706)$ or trimodality therapy $(n=177)$ including chemotherapy, radiation, and surgery. Pathological complete response rate following neoadjuvant therapy was obtained. Univariate and multivariate logistic regression analyses were performed to generate hazard ratios (HR) investigating factors associated with overall survival. Kaplan-Meier (K-M) method was used to estimate overall surviving proportion at 5 and 10 years.
\end{abstract}

Results: The K-M estimated 5 and 10 year overall survival for stage I disease was $71.3 \%$ and $57.8 \%$, respectively; stage II disease was $57.0 \%$ and $38.9 \%$, respectively; stage III disease was $57.8 \%$ and $41.5 \%$, respectively. On multivariate analysis, higher cT category $(P<0.001)$ resulted in worse survival. For locally advanced tumors (subcohort B), there was no significant difference in survival between chemoradiation alone compared to trimodality therapy ( $P=0.909$ on multivariate analysis).

Conclusions: Most providers manage locally advanced SCC of the rectum similar to anal cancer, which results in equivalent overall survival and spares patients from the additional morbidity associated with surgical resection.

\section{K E Y W O R D S}

cancer, chemoradiation, National Cancer Database, radiation, rectal, squamous

\section{1 | INTRODUCTION}

Squamous cell carcinoma (SCC) of the gastrointestinal (GI) tract most commonly occurs in the esophagus or anal

Author responsible for statistical analyses: SWD. canal, and prior studies report a $<1 \%$ incidence within the rectum. ${ }^{1}$ Due to its rarity, the etiology of SCC of the rectum remains unclear, although it has been linked to chronic inflammation and prior radiotherapy. ${ }^{2-5} \mathrm{~A}$ recent Surveillance, Epidemiology, and End Results (SEER)

This is an open access article under the terms of the Creative Commons Attribution License, which permits use, distribution and reproduction in any medium, provided the original work is properly cited.

(C) 2018 The Authors. Cancer Medicine published by John Wiley \& Sons Ltd. 
analysis showed those with SCC to have a favorable prognosis compared to adenocarcinoma of the rectum. ${ }^{6}$ While small, noninvasive tumors within the rectum can be managed with conservative measures such as surgery alone, more advanced rectal tumors often benefit from further intervention, including chemotherapy and/or radiation. ${ }^{7}$ Currently, no consensus guidelines exist for the treatment of nonmetastatic rectal cancer with SCC histology, which may be misguided considering its optimal treatment may differ from adenocarcinoma of the rectum. For example, the National Comprehensive Cancer Network recognizes mucosal melanoma of the GI tract as a separate entity entirely. ${ }^{8}$

Additionally, treatment of SCC of the anal canal has been shown to be managed markedly differently from rectal adenocarcinoma, with combined intensive chemotherapy and radiation without planned surgery being standard of care for locoregional anal SCC tumors, as reported by Nigro et al. ${ }^{9}$ The purpose of this study was to leverage the National Cancer Database (NCDB) to evaluate current trends in management and their effect on survival for this uncommon tumor. While the NCDB lacks local recurrence rates, unsalvageable recurrences result in reduced survival. The large patient numbers available with NCDB analysis should allow us to determine whether any survival detriment based on treatment allocation exists. $^{10}$

\section{2 | METHODS AND MATERIALS}

\subsection{Data source}

The NCBD, established in 1989, is a nationally recognized clinical oncology database sponsored by the American College of Surgeons and the American Cancer Society. The NCDB collects data from more than 1500 facilities accredited by the Commission on Cancer and contains information on treatments and outcomes for patients with malignant disease. The current database gathers more than $70 \%$ of new cancer diagnoses in the United States and contains more than 34 million historical records. ${ }^{11}$

Data were obtained from the NCDB for patients diagnosed with rectal cancer between 2004 and 2014 (264, 184 patients). We limited patients to squamous cell histology (histology codes 8070-8083, 258636 patients excluded). Patients with incomplete staging information or metastatic disease were excluded (2664 excluded). We excluded patients who died within 3 months of diagnosis due to competing risks of noncancer-related deaths (eg myocardial infarction; 429 excluded). Patient with unknown receipt of chemotherapy, radiation, or surgery were also excluded (215 excluded). While specific surgical technique is unavailable, surgery, if performed, was defined by the NCDB as definitive. Figure 1 shows the complete selection diagram with 261888 total patients excluded.

264184 evaluable patients with rectal tumors in the National Cancer Database

Non-squamous cell histology $=258228$

Tx, T0, Tis, Nx, Mx, M1 disease $=2664$

Died $<3$ months after diagnosis $=429$

Unknown treatment information $=215$ Total excluded $=261,888$

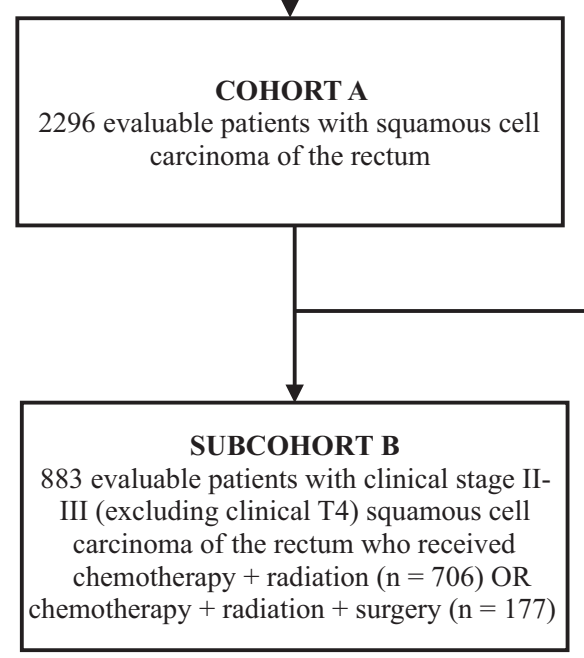

Clinical T1-T2, N0 disease $=955$

Clinical T4 disease $=288$ No therapy $=56$

Chemotherapy alone $=18$

Radiotherapy alone $=52$

Surgery alone $=21$

Chemotherapy and surgery $=13$

Radiation and surgery $=10$

Total excluded $=1,413$ 
The remaining 2296 patients, defined as cohort A, included cT1-4, cN0-2, cM0 SCC rectal tumors, according to American Joint Committee on Cancer (AJCC); these patients were then analyzed based on available database information. ${ }^{12}$ An additional subgroup, called subcohort $\mathrm{B}$, was further filtered to only include cT1-T3, cN+ or cT3, cN any SCC rectal tumors, which represent locally advanced tumors that, under current rectal cancer guidelines, include trimodality therapy as standard of care (chemotherapy, radiation, and surgery). ${ }^{8}$ To compare modalities, the two most common treatment approaches were included in the final analysis of subcohort B: chemotherapy and radiation or trimodality (chemotherapy, radiation therapy, and surgical resection). Patients with clinical T4 tumors were excluded from cohort B as those rectal tumors are less amenable to resection and can vary in their treatment sequence (eg neoadjuvant chemotherapy followed by restaging, then definitive local therapy). ${ }^{8,13}$

\section{2 | Statistical analyses}

The primary outcome measure of this study for each cohort was the overall survival of patients with nonmetastatic squamous cell carcinoma of the rectum. Important prognostic factors that may influence treatment or outcome, including gender, age, race, median income of zip code, distance to hospital, facility type, Charlson/Deyo score, tumor category, nodal category, receipt of chemotherapy, receipt of radiation, and receipt of surgery, were evaluated. A secondary outcome measure was pathological complete response rate among patients in each cohort.

Univariate and multivariate analyses (log rank, Cox regression, and binary logistic models) were performed to generate hazard ratios (HR) to investigate factors associated with overall survival. Potential prognostic variables in the multivariate models were chosen through purposeful selection and univariate analyses to investigate significance. Factors associated with a $P<0.10$ on univariate analysis were included in the multivariate models. Kaplan-Meier was used to estimate survival at 5 and 10 years. All statistical analyses were performed using the SPSS program (SPSS, version 24.0; SPSS Inc, Chicago, IL), and $P<0.05$ on multivariate analysis was considered statistically significant.

\section{3 | RESULTS}

\section{1 | Clinical and treatment characteristics of cohort A (cT1-T4, cN0-N2, cM0 SCC of the rectum)}

After planned exclusions, 2296 patients with nonmetastatic SCC of the rectum were identified from the NCDB database (Figure 1). Table 1 describes the clinical characteristics of cohort A. There was a strong female predilection
(70.8\% female). Most patients identified themselves as Caucasian (81.6\%), followed by African American (10.3\%) and Hispanic (5.7\%). The majority of patients had few comorbidities (Charlson/Deyo score $=0,81.2 \%)$. Most patients were managed through a comprehensive community cancer program (43.3\%). The remaining patients underwent therapy at academic or research facilities (30.8\%), integrated network cancer programs $(12.2 \%)$, and community cancer programs $(11.1 \%)$. The majority of patients had cT3 (35.8\%) and cN0 $(66.9 \%)$ disease. Regarding AJCC 7th edition staging for rectal cancer, 955 (41.6\%) were stage I, $582(25.3 \%)$ were stage II, and $759(33.1 \%)$ were stage III. There was an increase in recorded diagnoses over time, from 149 cases to in 2004 to 307 cases in 2014. The majority of patients received chemotherapy $(79.8 \%)$ and/or radiation $(82.1 \%)$, and only $27.3 \%$ of patients underwent surgery.

\section{2 | Overall survival analysis of cohort $\mathrm{A}$ (cT1-T4, cN0-N2, cM0 SCC of the rectum)}

Univariate and multivariate analyses were performed to investigate factors associated with overall survival, for which the complete results are shown in Table 2. On univariate analysis of patient characteristics, patients with increasing age, male gender, African American race, higher Charlson/ Deyo score, and lower median income of zip code were all associated with worse overall survival $(P<0.05)$. On multivariate analysis of patient characteristics, increasing age $(P<0.001)$, male gender (HR $=0.639$ for females, compared to male, $P<0.001)$, and higher Charlson/Score $(P<0.05)$ remained significant.

Regarding disease characteristics, higher cT category $(P<0.001)$ and $\mathrm{cN}$ category $(P=0.049)$ were associated with worse survival on univariate analysis. Notably, tumor grade did not influence survival. On multivariate analysis, only higher cT category remained significant $(\mathrm{HR}=1.551$ and 2.561 for cT3 and cT4 tumors compared to cT1, respectively, $P<0.001$ for each). Figure 2 shows the unadjusted Kaplan-Meier curve for each stage of disease, per AJCC 7th edition staging criteria. The Kaplan-Meier estimated 5- and 10-year survival for stage I disease was $71.3 \%$ and $57.8 \%$. The Kaplan-Meier estimated 5- and 10-year survival for stage II disease was $57.0 \%$ and $38.9 \%$. The Kaplan-Meier estimated 5- and 10-year survival for stage III disease was 57.8\% and $41.5 \%$, respectively. Notably, while there was a difference in survival from stage I to stage II/III disease (log rank $P<0.001$ for each), no survival difference was seen between stage II and stage III disease ( $\log \operatorname{rank} P=0.119)$, likely due to no difference in survival among nodal category with SCC histology.

Regarding treatment characteristics, all treatment modalities (chemotherapy, radiotherapy, and surgery) were associated with improved survival on univariate analysis. On 
TA B L E 1 Clinical and treatment characteristics of 2296 patients (cohort A) with nonmetastatic squamous cell cancer of the rectum in the National Cancer Database (2004-2014)

\begin{tabular}{|c|c|c|}
\hline & n or median & $\%$ or range \\
\hline \multicolumn{3}{|l|}{ Clinical characteristics } \\
\hline Age (years old, median) & 60 & $20-90$ \\
\hline \multicolumn{3}{|l|}{ Sex } \\
\hline Male & 670 & 29.2 \\
\hline Female & 1626 & 70.8 \\
\hline \multicolumn{3}{|l|}{ Race } \\
\hline White & 1874 & 81.6 \\
\hline African American & 237 & 10.3 \\
\hline American Indian & 4 & 0.2 \\
\hline Asian/Pacific Islander & 21 & 0.9 \\
\hline Unknown & 30 & 1.3 \\
\hline Hispanic & 130 & 5.7 \\
\hline \multicolumn{3}{|l|}{ Charlson/Deyo score } \\
\hline 0 & 1865 & 81.2 \\
\hline 1 & 294 & 12.8 \\
\hline 2 & 60 & 2.6 \\
\hline 3 & 77 & 3.4 \\
\hline \multicolumn{3}{|l|}{ Median income of zip } \\
\hline$<\$ 38000$ & 464 & 20.2 \\
\hline$\$ 38000-\$ 47999$ & 532 & 23.2 \\
\hline$\$ 48000-\$ 62999$ & 619 & 27.0 \\
\hline$\geq \$ 63000$ & 659 & 28.7 \\
\hline \multicolumn{3}{|l|}{ Distance to Hospital } \\
\hline$<25$ miles & 1880 & 81.9 \\
\hline 25-100 miles & 326 & 14.2 \\
\hline$>100$ miles & 68 & 3.0 \\
\hline \multicolumn{3}{|l|}{ Facility type } \\
\hline $\mathrm{CCP}$ & 254 & 11.1 \\
\hline $\mathrm{CCCP}$ & 995 & 43.3 \\
\hline Academic/research & 708 & 30.8 \\
\hline INCP & 281 & 12.2 \\
\hline \multicolumn{3}{|l|}{ Year of diagnosis } \\
\hline 2004 & 149 & 6.5 \\
\hline 2005 & 120 & 5.2 \\
\hline 2006 & 137 & 6.0 \\
\hline 2007 & 183 & 8.0 \\
\hline 2008 & 190 & 8.3 \\
\hline 2009 & 218 & 9.5 \\
\hline 2010 & 216 & 9.4 \\
\hline 2011 & 254 & 11.1 \\
\hline 2012 & 266 & 11.6 \\
\hline 2013 & 256 & 11.1 \\
\hline
\end{tabular}

(Continues)
TABLE 1 (Continued)

\begin{tabular}{|c|c|c|}
\hline & n or median & $\%$ or range \\
\hline 2014 & 307 & 13.4 \\
\hline \multicolumn{3}{|l|}{ Disease characteristics } \\
\hline \multicolumn{3}{|l|}{ cTcategory } \\
\hline cT1 & 592 & 25.8 \\
\hline cT2 & 595 & 25.9 \\
\hline cT3 & 821 & 35.8 \\
\hline cT4 & 288 & 12.5 \\
\hline \multicolumn{3}{|l|}{$\mathrm{cN}$ category } \\
\hline cNO & 1537 & 66.9 \\
\hline $\mathrm{cN} 1$ & 567 & 24.7 \\
\hline $\mathrm{cN} 2$ & 192 & 8.4 \\
\hline \multicolumn{3}{|l|}{ Clinical stage } \\
\hline I & 955 & 41.6 \\
\hline II & 582 & 25.3 \\
\hline III & 759 & 33.1 \\
\hline \multicolumn{3}{|l|}{ Tumor grade } \\
\hline Well differentiated & 121 & 5.3 \\
\hline $\begin{array}{l}\text { Moderately } \\
\text { differentiated }\end{array}$ & 757 & 33.0 \\
\hline Poorly differentiated & 767 & 33.4 \\
\hline Undifferentiated & 26 & 1.1 \\
\hline Unknown & 625 & 27.2 \\
\hline \multicolumn{3}{|l|}{ Treatment characteristics } \\
\hline \multicolumn{3}{|l|}{ Chemotherapy } \\
\hline No & 464 & 20.2 \\
\hline Yes & 1832 & 79.8 \\
\hline \multicolumn{3}{|l|}{ Radiotherapy } \\
\hline No & 411 & 17.9 \\
\hline Yes & 1885 & 82.1 \\
\hline \multicolumn{3}{|l|}{ Surgery } \\
\hline No & 1670 & 72.7 \\
\hline Yes & 626 & 27.3 \\
\hline
\end{tabular}

$\mathrm{CCCP}$, comprehensive community cancer program; $\mathrm{CCP}$, community cancer center; CI, confidence interval; HR, hazard ratio; INCP, integrated network cancer program.

multivariate, only receipt of chemotherapy $(\mathrm{HR}=0.531$, $P<0.001$ ) and surgery (HR $=0.758, P=0.002$ ) were associated with improved overall survival for cohort A.

\section{3 | Overall survival analysis of subcohort $\mathrm{B}$ (cT1-T2, cN+, cM0 or cT3, cN any, cM0 $\mathrm{SCC}$ of the rectum)}

To better clarify the optimal management of locally advanced tumors where the current standard of care for rectal cancer includes trimodality therapy, patients were filtered to only 
T A B L E 2 Analysis of factors associated with overall survival following diagnosis for 2296 patients (cohort A) with nonmetastatic squamous cell cancer of the rectum in the National Cancer Database (2004-2014)

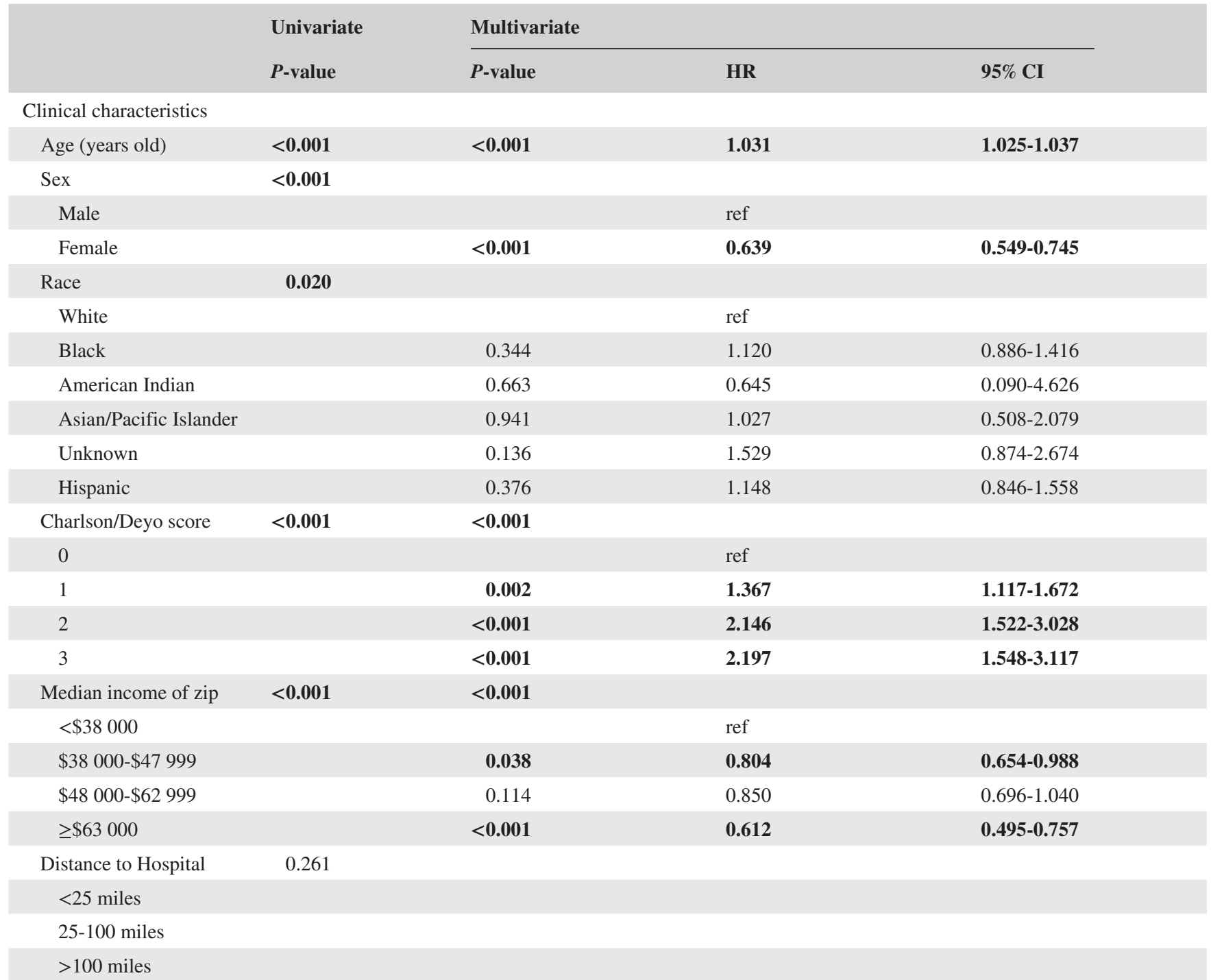

Facility type

0.927

CCP

CCCP

Academic/Research

INCP

Disease characteristics

\begin{tabular}{|c|c|c|c|c|}
\hline cTcategory & $<0.001$ & $<0.001$ & & \\
\hline $\mathrm{cT} 1$ & & & ref & \\
\hline $\mathrm{cT} 2$ & & 0.117 & 0.829 & $0.655-1.048$ \\
\hline cT3 & & $<0.001$ & 1.551 & 1.258-1.912 \\
\hline cT4 & & $<0.001$ & 2.561 & 2.019-3.249 \\
\hline $\mathrm{cN}$ category & 0.049 & 0.088 & & \\
\hline $\mathrm{cNO}$ & & & ref & \\
\hline $\mathrm{cN} 1$ & & 0.063 & 1.184 & $0.991-1.415$ \\
\hline $\mathrm{cN} 2$ & & 0.060 & 1.290 & $0.989-1.682$ \\
\hline
\end{tabular}

Tumor grade

0.240 
TABLE 2 (Continued)

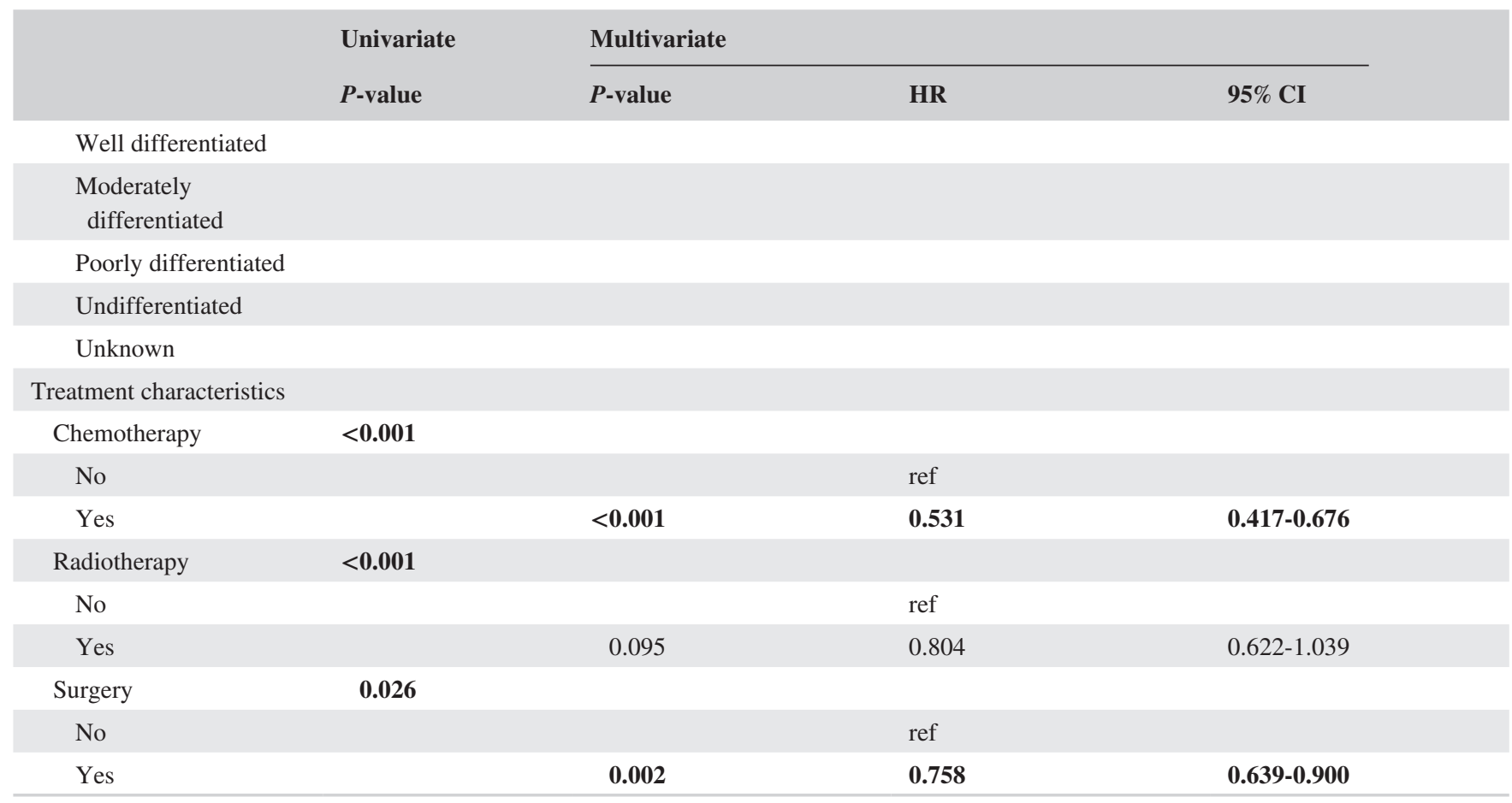

$\mathrm{CCCP}$, comprehensive community cancer program; CCP, community cancer center; CI, confidence interval; HR, hazard ratio; INCP, integrated network cancer program; RT, radiation therapy.

Numbers bolded for $P<0.05$.

include clinical stage II and III disease (excluding cT4) which resulted in 1053 patients. Among these patients, the majority either received chemotherapy and radiation $(n=706,67 \%)$ or chemotherapy, radiation, and surgery $(\mathrm{n}=177,16.8 \%)$. Less common combined modalities utilized were chemotherapy and surgery $(\mathrm{n}=13,1.2 \%)$ and radiation and surgery $(\mathrm{n}=10,0.9 \%)$. To compare the two most common modalities, other regimens were excluded from the final subcohort B survival analysis (Figure 1), resulting in 883 patients with complete evaluable treatment information (Table 3).

On univariate analysis of subcohort B, younger age, female gender, lower Charlson-Deyo score, lower cT category, and trimodality therapy (versus chemotherapy and radiation) were associated with improved overall survival $(P<0.05$ for each). On multivariate analysis, older age (HR $=1.019$ per year, $P=0.001)$, higher Charlson/Deyo scores $(P<0.05)$, and lower median income of zip $(P=0.001)$ were associated with worse survival. Of note, therapy type, comparing chemotherapy, and radiation to trimodality therapy (chemotherapy, radiation, and surgery) showed no significant difference in survival $(P=0.909$ on multivariate analysis). Figure 3 shows the unadjusted Kaplan-Meier curve for subcohort B, separated by therapy type. The Kaplan-Meier estimated 5and 10-year survival for entire cohort B is $63.8 \%$ and $50.3 \%$ (log rank $P=0.936$ between receipt of chemotherapy and radiation \pm surgery).

\section{4 | Pathological response to therapy}

Among cohort $\mathrm{A}$, who underwent radiation therapy prior to surgery and have available pathology information, $36.0 \%$ (41/114 patients) experienced a complete pathological response. 39/41 (95.1\%) of those patients with a complete pathological response also received neoadjuvant chemotherapy prior to surgery, in addition to radiation. The median number of days from end of radiation to definitive surgery for those with a complete pathological response (p0) was 67 days $(n=38$, range 9-237 days) versus 73 days ( $\mathrm{n}=70$, range 6-320 days) for those without a complete response (p1-4 disease) on surgical pathology $(P=0.29)$. The median regional radiation dose was $45 \mathrm{~Gy}$ with a boost administered to $46.3 \%$ of patients (median $5.4 \mathrm{~Gy}$ ). While univariate analysis demonstrated a trend toward a survival advantage with complete pathological responders $(P=0.085)$, it was not significant on multivariate analysis $(P=0.110)$.

Among patients in subcohort B who underwent radiation therapy prior to surgery and have available pathology information, $41.1 \%$ (30/73 patients) experienced a complete pathological response on surgical pathology. No survival advantage was observed comparing pathologic complete versus noncomplete responders in subcohort B $(P=0.129$ on univariate analysis). 


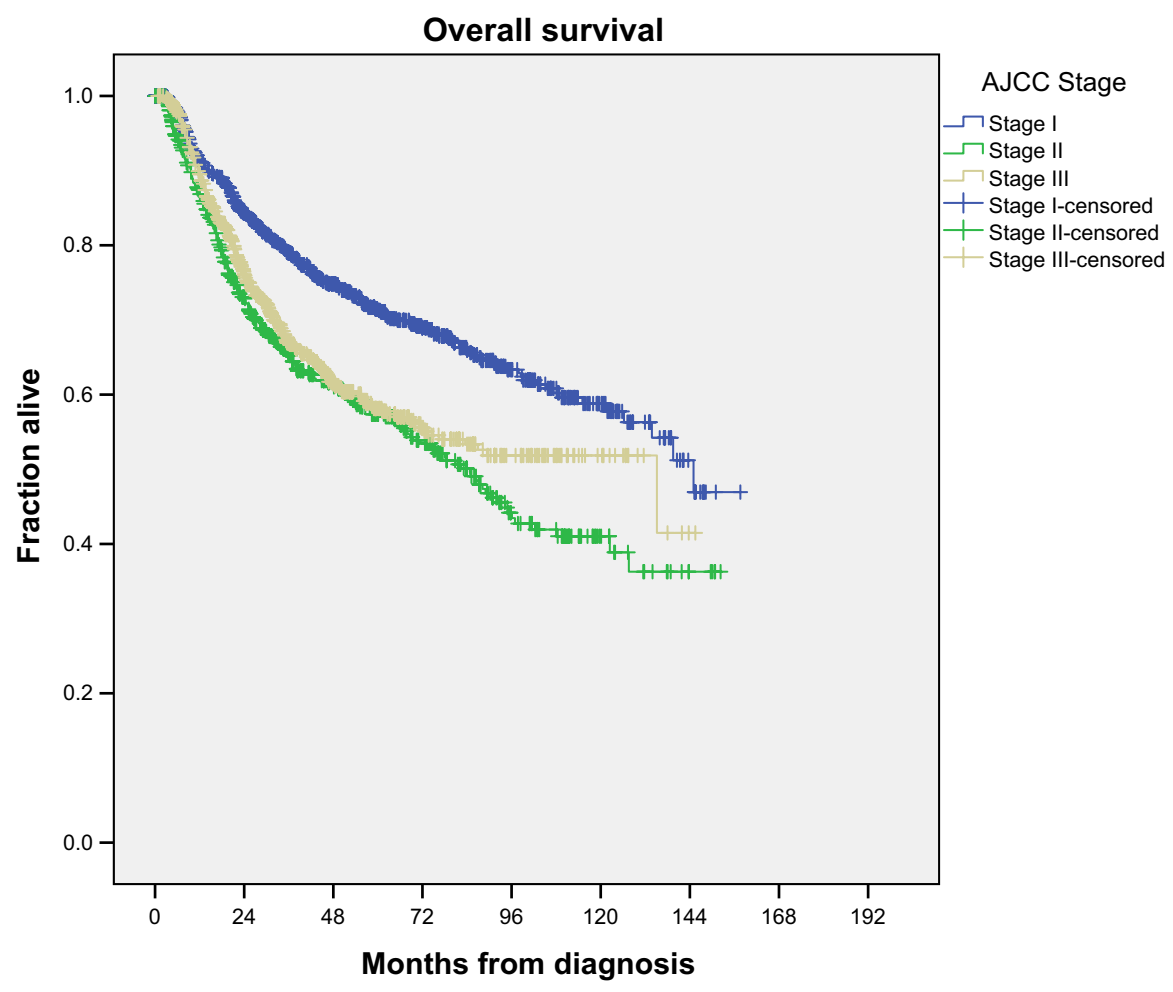

F I G U R E 2 Unadjusted Kaplan-Meier curve for each stage of disease within cohort A, per American Joint Committee on Cancer 7 th edition staging criteria to tumor category, consistent with a prior review. ${ }^{15}$ Reasons we hypothesize for this finding include difficulty in accurately assessing nodal status clinically, unique history of SCC of the rectum nodal metastases, or treatment strategies currently in use are effective in controlling nodal disease with SCC histology.

We specifically created subcohort B that, under rectal cancer guidelines, may be managed with trimodality therapy, which is the current standard of care. However, our study supports the effectiveness of chemoradiation, without surgery. The NCDB (and SEER analyses) are unable to detect rates of local recurrence and salvage surgery for those who do not receive surgery as part of their initial treatment course. Regardless, even if a substantial portion of patients ultimately require salvage surgery, our data do not show a survival benefit to adding surgery during the initial course. This is consistent with a prior report by Kulaylat et al who grouped stage I-III patients and found that those with SCC of the anus requiring salvage surgery (ie $\geq 12$ weeks after chemoradiation) had worse survival, compared to no survival difference for those with SCC of the rectum receiving salvage surgery. Taken in conjunction with the present study, planned or late surgery for SCC of the rectum results in no survival benefit after chemoradiation. ${ }^{16}$

While the NCDB does not provide specific chemotherapy agents, there is a growing body of literature from individual institutions showing good outcomes with agents used for the treatment of anal SCC. ${ }^{17}$ Clark et al reported seven cases treated using the Anal Cancer Trial II (ACT II) protocol, which included 5.5 weeks of radiation with concurrent 
TA B L E 3 Analysis of factors associated with overall survival following diagnosis for 883 patients (subcohort B) with locally advanced (cT1-T2, cN+, cM0 or cT3, cN any, cM0) squamous cell cancer of the rectum in the National Cancer Database (2004-2014)

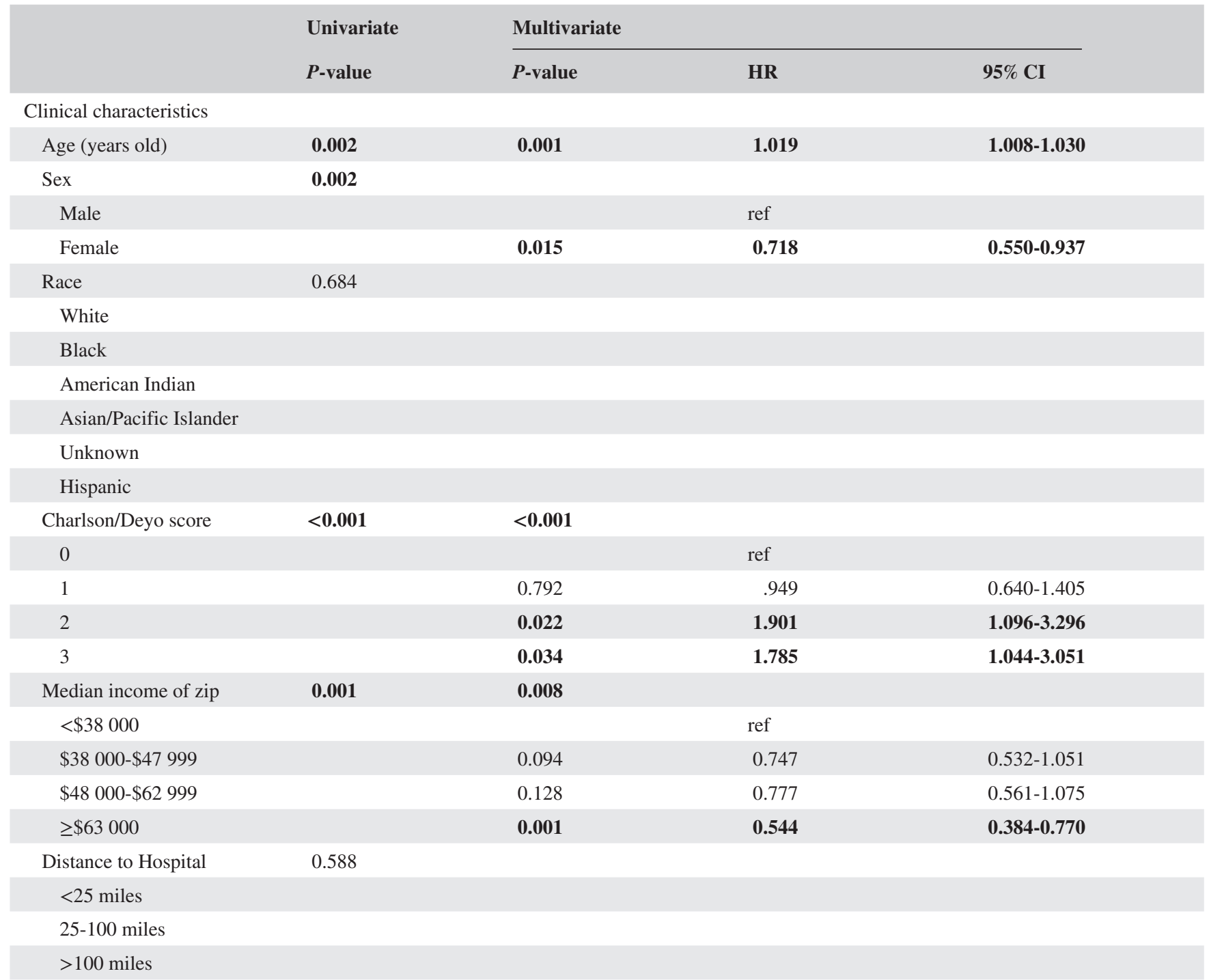

Facility type

0.682

$\mathrm{CCP}$

$\mathrm{CCCP}$

Academic/Research

INCP

Disease characteristics

cTcategory

0.001

cT1

cT2

0.723

ref

cT3

0.174

0.877

0.424-1.814

$\mathrm{cN}$ category

0.375

cNO

$\mathrm{cN} 1$

$\mathrm{cN} 2$

Tumor grade

0.353

Well differentiated 
T A B L E 3 (Continued)

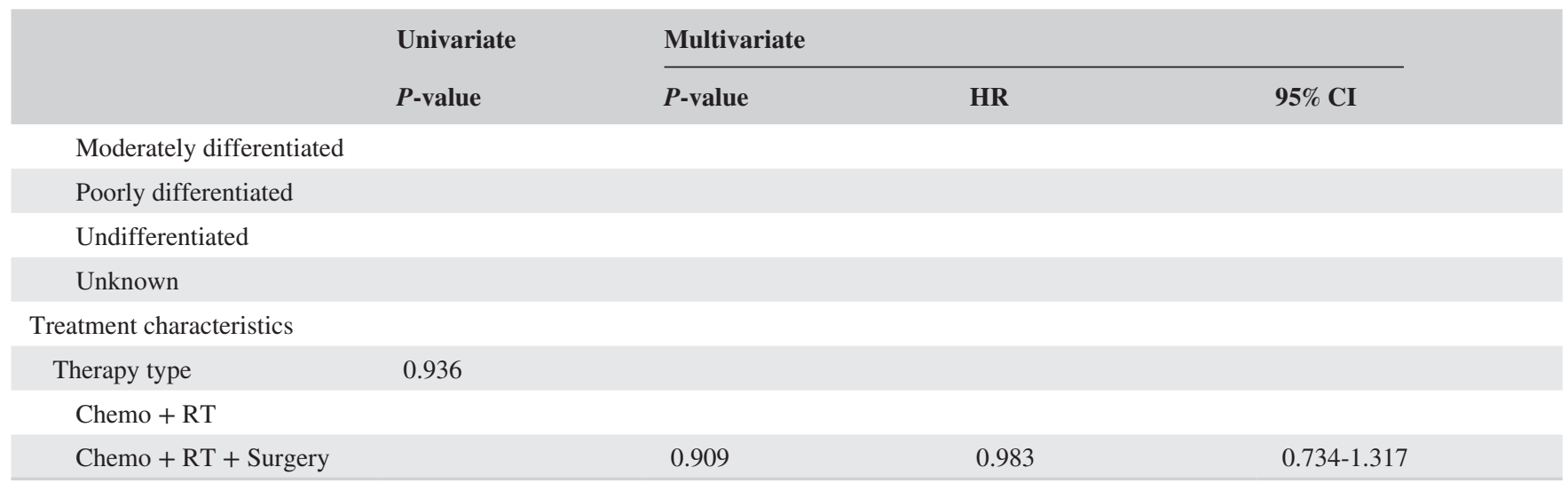

$\mathrm{CCCP}$, comprehensive community cancer program; CCP, community cancer center; CI, confidence interval; HR, hazard ratio; INCP, integrated network cancer program; RT, radiation therapy.

Numbers bolded for $P<0.05$.

F I G U RE 3 Unadjusted Kaplan-

Meier curve of overall survival from time of diagnoses for patients receiving either chemotherapy and radiation or trimodality therapy (chemotherapy, radiation, and chemotherapy) for locally advanced squamous cell cancer of the rectum (subcohort B). No survival difference was observed $(P=0.304$ by log rank)

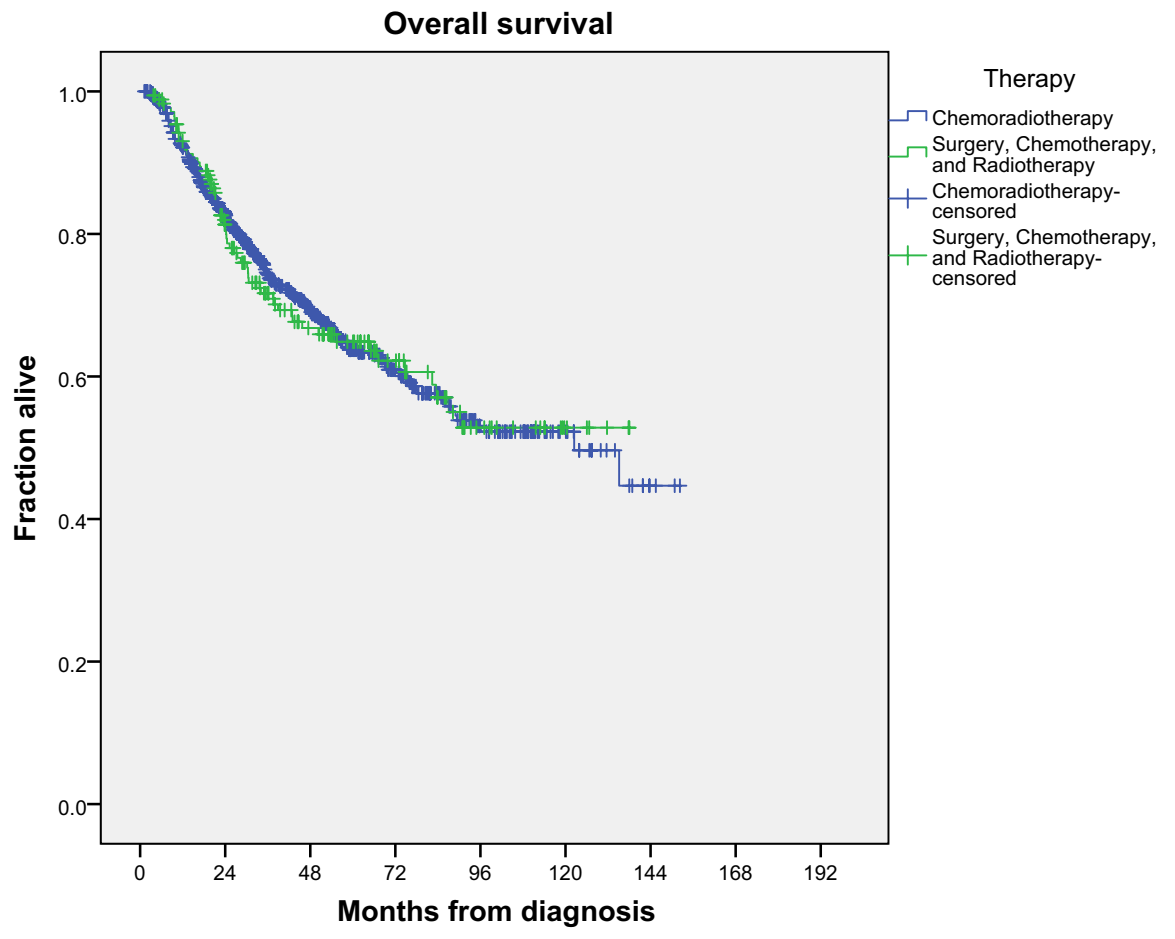

5-flurouracil and mitomycin $\mathrm{C}$ or cisplatin. ${ }^{18,19}$ At a median follow-up of 18 months, no recurrences were noted, with only one patient undergoing surgery, which showed no viable tumor on surgical pathology. Sturgeon et $\mathrm{al}^{20}$ reported outcomes for 14 patients with SCC of the rectum who underwent similar definitive chemoradiation using an anal cancer regimen. With a median follow-up of 4.5 years, only $3 / 14$ patients recurred, and two were successfully managed with salvage surgery. Musio et $\mathrm{al}^{21}$ reported outcomes for eight patients treated with definitive management similar to anal cancer; their study reported a negative biopsy in $75 \%$ of patients 6 months after chemoradiation, and only one patient required a salvage surgery at the end of treatment. For those that received surgery, our study reports a short interval between radiation and surgery (median 69 days for cohort A), indicating that the surgery was planned and not performed as salvage. Additional time from completion of radiation may have resulted in higher pathological complete response rates, with data from the ACT II trial suggesting that 182 days is the optimal time to assess response in anal SCC. ${ }^{22}$ Unfortunately, surveillance for rectal SCC would involve endoscopy to evaluate tumor response, whereas anal cancer can be more easily examined with inspection or digital rectal exam. Also, proximal versus distal tumor location is not provided by the NCDB, limiting further analysis. However, it should be noted that the normal tissue (ie skin) around the anus is more sensitive to radiation, and it may be possible to treat less distal tumors with higher doses of radiation to gross disease, 
further improving the tumor response rate. ${ }^{23}$ Most patients in our series received a moderate dose of 45-50.4 Gy.

In summary, existing literature shows that chemotherapy regimens utilized for anal cancer (eg 5-flurouracil and mitomycin C) given concurrently with radiation provides a durable response and that patients do well with salvage surgery, if needed. This is exemplified with our data, showing similar survival for patients with stage II-III (excluding T4) cancer managed with chemoradiation. While our NCDB analysis does not provide chemotherapy agents used, the current literature supports regimens used in anal cancer. ${ }^{24}$ One hypothesis for the lower pathological complete response rate in our study compared to institutional reports is that more effective, albeit more toxic, regimens used in anal cancer (eg mitomycin C) given concurrently with radiation may have been substituted with a more tolerable concurrent chemotherapy agent (eg capecitabine), with the expectation that the patient will undergo definitive resection.

Limitations to our study should be noted. As discussed, the NCDB is limited by nonrandom allocation to treatment modality and the lack of detailed information regarding medical comorbidities, both of which may obscure comparisons of treatment methods through the introduction of confounding. We performed multivariate analysis to adjust for measured potential confounders, but significant potential biases remain. Furthermore, chemotherapy agents and extent (ie number of cycles) is not known. However, we excluded patients with incomplete staging and unknown treatment information, therefore strengthening the reliability of the outcome for each treatment group. Lastly, disease-specific outcomes including local recurrence and salvage surgery rate is unknown.

\section{\begin{tabular}{l|l}
5 & CONCLUSION
\end{tabular}}

This database analysis shows that most providers are managing locally advanced rectal SCC similar to anal cancer, which results in equivalent overall survival and spares patients from the additional morbidity associated with surgical resection. While most surgeries were presumably planned, a pathological complete response rate up to $41 \%$ suggests radiation and chemotherapy has an effective response.

\section{CONFLICT OF INTEREST}

None.

\section{ORCID}

Sunil W. Dutta iD https://orcid.org/0000-0003-3574-6569

Daniel M. Trifiletti iD https://orcid.org/0000-0002-9150-2916

\section{REFERENCES}

1. Kang H, O'Connell JB, Leonardi MJ, et al. Rare tumors of the colon and rectum: a national review. Int $J$ Colorectal Dis. 2007;22(2):183-189.

2. Scaringi S, Bisogni D, Messerini L, et al. Squamous cell carcinoma of the middle rectum: report of a case and literature overview. Int J Surg Case Rep. 2015;7C:127-129.

3. Leung KK, Heitzman J, Madan A. Squamous cell carcinoma of the rectum 21 years after radiotherapy for cervical carcinoma. Saudi J Gastroenterol. 2009;15(3):196-198.

4. Cheng H, Sitrin MD, Satchidanand SK, et al. Colonic squamous cell carcinoma in ulcerative colitis: report of a case and review of the literature. Can J Gastroenterol. 2007;21(1):47-50.

5. Zirkin RM, McCord DL. Squamous cell carcinoma of the rectum: report of a case complicating chronic ulcerative colitis. Dis Colon Rectum. 1963;6:370-373.

6. Chiu MS, Verma V, Bennion NR, et al. Comparison of outcomes between rectal squamous cell carcinoma and adenocarcinoma. Cancer Med. 2016;5(12):3394-3402.

7. Glimelius B, Tiret E, Cervantes A, et al. Rectal cancer: ESMO clinical practice guidelines for diagnosis, treatment and followup. Ann Oncol. 2013;24(suppl 6):vi81-vi88.

8. National Comprehensive Cancer Network.Rectal cancer (Version 1.2018). https://www.nccn.org/professionals/physician_gls/pdf/ rectal.pdf. Accessed March 13, 2018; April 2, 2018.

9. Nigro ND, Seydel HG, Considine B, et al. Combined preoperative radiation and chemotherapy for squamous cell carcinoma of the anal canal. Cancer. 1983;51(10):1826-1829.

10. Cai Y, Li Z, Gu X, et al. Prognostic factors associated with locally recurrent rectal cancer following primary surgery (Review). Oncol Lett. 2014;7(1):10-16.

11. American College of Surgeons, Q.P.National cancer database. https://www.facs.org/quality-programs/cancer/ncdb. Accessed December 7, 2017.

12. American College of Surgeons. National cancer database: patient user file data dictionary items. http://ncdbpuf.facs. org/?q=node/259. Accessed December 7, 2017.

13. Wu JS. Rectal cancer staging. Clin Colon Rectal Surg. 2007;20(3):148-157.

14. Bokey EL, Chapuis PH, Hughes WJ, et al. Morbidity, mortality and survival following resection for carcinoma of the rectum at Concord Hospital. Aust N Z J Surg. 1990;60(4):253-259.

15. Kassir R, Baccot S, Bouarioua N, et al. Squamous cell carcinoma of middle rectum: literature review. Int J Surg Case Rep. 2014;5(2):86-90.

16. Kulaylat AS, Hollenbeak CS, Stewart DB Sr. Squamous cancers of the rectum demonstrate poorer survival and increased need for salvage surgery compared with squamous cancers of the anus. Dis Colon Rectum. 2017;60(9):922-927.

17. Ghosn M, Kourie HR, Abdayem P, et al. Anal cancer treatment: current status and future perspectives. World J Gastroenterol. 2015;21(8):2294-2302.

18. James RD, Glynne-Jones R, Meadows HM, et al. Mitomycin or cisplatin chemoradiation with or without maintenance chemotherapy for treatment of squamous-cell carcinoma of the anus (ACT II): a randomised, phase 3, open-label, 2 × 2 factorial trial. Lancet Oncol. 2013;14(6):516-524.

19. Clark J, Cleator S, Goldin R, et al. Treatment of primary rectal squamous cell carcinoma by primary chemoradiotherapy: should 
surgery still be considered a standard of care? Eur J Cancer. 2008;44(16):2340-2343.

20. Sturgeon JD, Crane CH, Krishnan S, et al. Definitive chemoradiation for squamous cell carcinoma of the rectum. Am J Clin Oncol. 2017;40(2):163-166.

21. Musio D, De Felice F, Manfrida S, et al. Squamous cell carcinoma of the rectum: the treatment paradigm. Eur J Surg Oncol. 2015;41(8):1054-1058.

22. Glynne-Jones R, Sebag-Montefiore D, Meadows HM, et al. Best time to assess complete clinical response after chemoradiotherapy in squamous cell carcinoma of the anus (ACT II): a post-hoc analysis of randomised controlled phase 3 trial. Lancet Oncol. 2017;18(3):347-356.

23. Gunther JR, Chadha AS, Shin US, et al. Preoperative radiation dose escalation for rectal cancer using a concomitant boost strategy improves tumor downstaging without increasing toxicity: a matched-pair analysis. Adv Radiat Oncol. 2017;2(3):455-464.

24. Glynne-Jones R, Nilsson PJ, Aschele C, et al. Anal cancer: ESMO-ESSO-ESTRO clinical practice guidelines for diagnosis, treatment and follow-up. Radiother Oncol. 2014;111(3):330-339.

\section{How to cite this article: Dutta SW, Alonso CE,} Waddle MR, Khandelwal SR, Janowski E-M, Trifiletti DM. Squamous cell carcinoma of the rectum: Practice trends and patient survival. Cancer Med.

2018;7:6093-6103. https://doi.org/10.1002/cam4.1893 